\title{
Sex differences and effects of prenatal exposure to excess testosterone on ventral tegmental area dopamine neurons in adult sheep
}

\author{
Erinna C. Z. Brown, ${ }^{1}$ Casey J. Steadman, ${ }^{2}$ Theresa M. Lee, ${ }^{3}$ Vasantha Padmanabhan, ${ }^{4}$ Michael N. Lehman ${ }^{5}$ and \\ Lique M. Coolen ${ }^{1,2,5}$ \\ ${ }^{1}$ Department of Anatomy and Cell Biology, The University of Western Ontario, London, ON N6A 5C1, Canada \\ ${ }^{2}$ Department of Physiology and Biophysics, University of Mississippi Medical Center, Jackson, MS 39216, USA \\ ${ }^{3}$ Department of Psychology, University of Tennessee Knoxville, Knoxville, TN, USA \\ ${ }^{4}$ Department of Pediatrics, University of Michigan, Ann Arbor, MI, USA \\ ${ }^{5}$ Department of Neurobiology and Anatomical Sciences, University of Mississippi Medical Center, Jackson, MS 39216, USA
}

Keywords: mesolimbic, midbrain, reward, sex dimorphism, steroid

\begin{abstract}
Prenatal testosterone $(T)$ excess in sheep results in a wide array of reproductive neuroendocrine deficits and alterations in motivated behavior. The ventral tegmental area (VTA) plays a critical role in reward and motivated behaviors and is hypothesised to be targeted by prenatal T. Here we report a sex difference in the number VTA dopamine cells in the adult sheep, with higher numbers of tyrosine hydroxylase (TH)-immunoreactive (-ir) cells in males than females. Moreover, prenatal exposure to excess $\mathrm{T}$ during either gestational days 30-90 or 60-90 resulted in increased numbers of VTA TH-ir cells in adult ewes compared to control females. Stereological analysis confirmed significantly greater numbers of neurons in the VTA of males and prenatal T-treated ewes, which was primarily accounted for by greater numbers of TH-ir cells. In addition, immunoreactivity for TH in the cells was denser in males and prenatal T-treated females, suggesting that sex differences and prenatal exposure to excess $\mathrm{T}$ affects both numbers of cells expressing TH and the protein levels within dopamine cells. Sex differences were also noted in numbers of THir cells in the substantia nigra, with more cells in males than females. However, prenatal exposure to excess $T$ did not affect numbers of TH-ir cells in the substantia nigra, suggesting that this sex difference is organised independently of prenatal actions of $\mathrm{T}$. Together, these results demonstrate sex differences in the sheep VTA dopamine system which are mimicked by prenatal treatment with excess $\mathrm{T}$.
\end{abstract}

\section{Introduction}

The effects of prenatal testosterone $(\mathrm{T})$ on reproductive physiology and behavior have been documented in several species including rat (Wolf et al., 2002; Wu et al., 2010), rhesus macaque (Goy et al., 1988; Abbott et al., 2005), human (Merke \& Bornstein, 2005) and sheep (Wood \& Foster, 1998; Robinson et al., 2002; Padmanabhan et al., 2006; Bormann et al., 2011; Hogg et al., 2011). For sheep, the critical period of sexual differentiation of the hypothalamic-pituitary-gonadal axis spans gestational days 30-90 of their 147-day gestational period (Clarke et al., 1976; Wood \& Foster, 1998; Robinson et al., 2002; Padmanabhan et al., 2006). Females treated with T during gestational days 30-90 are born with virilized genitalia (Wood \& Foster, 1998) and multiple reproductive neuroendocrine and ovarian disruptions (Wood \& Foster, 1998; Padmanabhan et al., 2006). Female sheep treated with $\mathrm{T}$ during gestational days

Correspondence: Lique M. Coolen, ${ }^{1}$ Department of Anatomy and Cell Biology, as above.

E-mail: 1coolen@umc.edu

Received 4 November 2014, revised 19 January 2015, accepted 10 February 2015
60-90 are born with normal feminine external genitalia (Steckler et al., 2007) and ovarian cycles in the first breeding season (Savabieasfahani et al., 2005). However, they display masculinized urinary posture, altered expression of genes coding for components of steroidogenesis in the developing ovary (Hogg et al., 2011), delayed estradiol-positive feedback response (Savabieasfahani et al., 2005; Steckler et al., 2007) and a masculinized sexually dimorphic nucleus of the preoptic area (Alexander et al., 2011; Roselli et al., 2011).

In addition, prenatal $\mathrm{T}$ excess treatment alters motivated behaviors. Sheep live in stable social groups where dominance hierarchies arise from the competition for resources (e.g. food and mates) and males are at the top of the dominance hierarchy. Prenatal $\mathrm{T}$ treatment significantly increases the ranking of females (Roberts et al., 2009) and masculinizes sexual behavior (Roberts et al., 2008, 2009). The mesolimbic system plays a critical role in motivated behaviors including food intake (Palmiter, 2007) and sexual behavior (Fields et al., 2007; Frohmader et al., 2010). The mesolimbic system consists of dopamine producing neurons located in the ventral tegmental area (VTA) and their projections (Fields et al., 2007). Studies in rodents showed that VTA dopamine neurons are sexually 
dimorphic (Engele et al., 1989; McArthur et al., 2007). However, to what extent prenatal steroid hormones contribute to the sexual dimorphism is unclear, as sex differences in midbrain dopamine neurons can occur in the absence of steroid hormones and may be caused by sex chromosome complement(s) (Engele et al., 1989; Carruth et al., 2002; Arnold, 2014). VTA dopamine neurons express androgen receptor (AR) (Kritzer, 1997) and estrogen receptor (ER) $\beta$ (Kritzer, 1997; Creutz \& Kritzer, 2002), and tyrosine hydroxylase $(\mathrm{TH})$, the rate-limiting enzyme of the dopamine biosynthesis pathway, can be activated by androgen (Jeong et al., 2006) and estrogen (Ivanova \& Beyer, 2003; Serova et al., 2004; Maharjan et al., 2005) receptor activation. Therefore, we hypothesise that excess prenatal $\mathrm{T}$ treatment alters the VTA dopamine neurons in the sheep mesolimbic system. To address this hypothesis, we characterised the distribution and presence of sex differences of midbrain dopamine neurons in male and female sheep, and examined the effect of prenatal $\mathrm{T}$ treatment of female sheep during gestational days $60-90$ or $30-90$ on the number and size of midbrain dopamine neurons.

\section{Materials and methods}

\section{Animals and general care}

Female and male Suffolk ewes were used for all experiments. All animals were raised at the Sheep Research Facility at the University of Michigan (Ann Arbor, MI, USA; $42^{\circ} 18^{\prime} \mathrm{N}$ ). Animal care, including husbandry and nutrition, were conducted as previously described (Manikkam et al., 2004; Jackson et al., 2013). Briefly, adult Suffolk ewes with proven fertility were purchased in the local Michigan area, housed under a natural photoperiod in the pasture, and groupfed daily. After birth, each mother and her lambs were individually housed for the first 3 days, then group-housed in a barn, and lambs were weaned at 8 weeks of age. After weaning, female lambs were kept in their same-age cohort, always able to freely interact with each other. All experimental procedures were approved by the University Animal Care and Use Committee at the University of Michigan and are consistent with National Research Council's Guide for the Care and Use of Laboratory Animals.

\section{Experimental groups}

Experimental females were exposed prenatally to excess $\mathrm{T}$ between either gestational days 60 and 90 (group T60-90) of the 147-day gestational period or, in a separate study, for a longer prenatal period between gestational days 30 and 90 (T30-90). These two experimental groups each had age-matched controls (C) that were reared and housed simultaneous with the corresponding T-treated groups (groups C60-90 and C30-90 respectively). For generation of prenatal T-treated females, pregnant Suffolk ewes of known conception dates received twice weekly $2-\mathrm{mL}$ intramuscular injections of $100 \mathrm{mg}$ testosterone propionate $(\sim 1.2 \mathrm{mg} / \mathrm{kg}$; Sigma-Aldrich Corp., St Louis, MO, USA) in cottonseed oil for 30 or 60 days, between gestational days 60 to 90 (T60-90) or 30 to 90 (T30-90) of the 147-day gestational term. The dose and mode of $\mathrm{T}$ propionate treatment has been shown to achieve a concentration of $\mathrm{T}$ similar to that in to adult males in pregnant ewes and levels of $\mathrm{T}$ in female fetuses that are similar to those of male fetuses $(\sim 0.6 \mathrm{ng} / \mathrm{mL}$ in the umbilical arterial blood; Veiga-Lopez et al., 2011). Control females did not receive vehicle, as previous studies have demonstrated no differences in reproductive attributes between vehicle-treated and nonvehicle controls in a previous study (Veiga-Lopez et al., 2008).
In order to control steroid levels between the groups of females, all ewes were ovariectomized at 2 years of age, were sequentially treated with progesterone implants (CIDR; Inter AG, Hamilton, Waikato, New Zealand) and estradiol (E2; Silastic implants; $16 \mathrm{~h}$ after removal of CIDR), in a regimen previously demonstrated to artificially mimic late follicular phase levels (Jackson et al., 2013). All animals were killed $20 \mathrm{~h}$ after E2 ( 2 months following ovariectomy) and the brains collected.

One group of control males was added for comparison of sex differences. These males were bred on the farm and did not receive any prenatal treatments. They were raised with either group of females, but were combined as one group and served as control Male group for both studies. These males were gonadally intact, vasectomised at 3.5 months, sexually experienced, and killed at 1012 months of age.

\section{Tissue collection}

Animals received two intravenous injections of heparin $(25000 \mathrm{U}$ given $10 \mathrm{~min}$ apart; Abraxiz Pharmaceutical Products, Schumberry, IL, USA), were anesthetised with an intravenous administration of sodium pentobarbital (2-3 g; Sigma-Aldrich), and were killed with decapitation. Each head was perfused via both internal carotids with $6 \mathrm{~L}$ of $4 \%$ paraformaldehyde in $0.1 \mathrm{M}$ sodium phosphate buffer ( $\mathrm{PB} ; \mathrm{pH} 7.3$ ) mixed with $0.1 \%$ sodium nitrate and $10 \mathrm{U} / \mathrm{mL}$ heparin. After perfusion, the brains were removed; a block of tissue containing the midbrain was dissected out and placed in $4 \%$ paraformaldehyde in $\mathrm{PB}$ overnight for postfixation at $4{ }^{\circ} \mathrm{C}$. The block was then transferred into $30 \%$ sucrose in $\mathrm{PB}$ at $4{ }^{\circ} \mathrm{C}$ to complete infiltration. Frozen coronal sections were cut into 10 parallel series $(45-\mu \mathrm{m}$-thick slices) using a freezing microtome (Microm HM400R; Walldorf, Germany) and stored in cryoprotective solution $(30 \%$ ethylene glycol, $0.1 \%$ sodium azide and $30 \%$ sucrose in $\mathrm{PB}$ ) at $-20{ }^{\circ} \mathrm{C}$ until being processed for immunohistochemistry.

\section{Immunohistochemistry general methods}

For each experiment, tissue sections from all groups were processed simultaneously as described below. All steps were performed at room temperature with gentle agitation. Free-floating sections were rinsed thoroughly in $0.1 \mathrm{M}$ phosphate-buffered saline (PBS) between incubations. Sections were incubated with $1 \% \mathrm{H}_{2} \mathrm{O}_{2}$ (Fisher Scientific, Pittsburgh, PA, USA) in PBS for $10 \mathrm{~min}$ to eliminate the endogenous peroxidase activity. Unless otherwise specified, tissue sections were blocked for $1 \mathrm{~h}$ with incubation solution containing PBS, $0.4 \%$ Triton X-100 (Sigma-Aldrich) and 4\% normal goat serum (Jackson ImmunoResearch Laboratories, West Grove, PA, USA), and all primary antibody incubations were performed overnight at room temperature in the incubation solution. After staining, the sections were washed several times with $0.1 \mathrm{M} \mathrm{PB}$, mounted on glass slides with $0.3 \%$ gelatin in $\mathrm{ddH}_{2} \mathrm{O}$, air-dried and coverslipped with DPX (Electron Microscopy Sciences, Fort Washington, PA, USA).

\section{Immunohistochemistry for TH}

Tissue sections from the midbrain containing the VTA were incubated overnight with monoclonal antibody to TH (1:50 000, MAB5280; Millipore/Chemicon International, Temecula, CA, USA) followed by 1 -h incubations in biotinylated goat anti-mouse IgG (1 : 500; Vector Laboratories, Burlingame, CA, USA) and avidin- 
horseradish peroxidopaminese complex (ABC-elite, $1: 500$ in $0.1 \mathrm{M}$ PBS; Vector Laboratories). TH immunoreactivity was visualised using diaminobenzidine (DAB; Sigma-Aldrich) in PB containing $0.012 \% \mathrm{H}_{2} \mathrm{O}_{2}$, resulting in a reddish-brown reaction product. Sections were counterstained using Cresyl Violet [0.625\% Cresyl Violet acetate (Sigma-Aldrich) and $0.0625 \%$ sodium acetate trihydrate (Fisher) in $\mathrm{ddH}_{2} \mathrm{O}$ ] only for stereological analysis and NEUROLUCIDA mapping.

\section{Antibody controls}

The antibody used for $\mathrm{TH}$ has been previously characterised for use in rodent brain (Balfour et al., 2004). Omission of the primary antibody prevented all staining, and Western blot analysis using sheep midbrain protein revealed a single band at the appropriate weight $(64 \mathrm{kDa})$. Finally, the distribution of the TH-immunoreactive (-ir) cells appeared similar to these described in other mammalian species (see Results and Discussion).

\section{NEUROLUCIDA mapping of TH-ir cell distribution}

The neuroanatomical localization of the TH-ir neurons was first analysed in control males $(n=8)$ and females (C60-90; $n=9)$. Using NEUROLUCIDA mapping software (MicroBrightfield Bioscience, Williston, VT, USA) and a CCD camera (Microfire; Optronics, Goleta, CA, USA) attached to a Leica microscope (DM5000B; Leica Microsystems, Wetzlar, Germany), images were taken and contour drawings were made for one representative control male and female (C60-90), using Cresyl Violet-counterstained sections. Contour drawings were made of three sections, representative of the rostral, middle and caudal levels in the VTA and substantia nigra (SN), and the locations of the TH-ir neurons were noted (Fig. 1). For the SN, the standard area of analysis was dorsal and lateral to the VTA and did not discriminate between the SN pars compacta and pars reticulata (Fig. 1). Major anatomical landmarks were used to delineate the VTA (based on delineations in rat VTA; Balfour et al., 2004), including the fasciculus retroflexus (FR), cerebral peduncle (CP), central aquaduct (AQ), dorsal tegmental bundle (DTB), posterior commissure (PC), red nucleus $(\mathrm{RN})$, central grey (CG), oculomotor nerve bundles (OM), oculomotor nucleus (ON) (Fig. 1). The medial tip of the $\mathrm{CP}$ was defined as the boundary between the VTA and SN. The rostral level of the VTA was characterised as having the FR dorsal to the VTA. The FR then descends ventrally as the VTA moves more caudally (Fig. 1A-D). The middle level of the VTA was determined as the level at which the RN appears and there are abundant numbers of OMs (Fig. 1E-H). At what was determined as the caudal level of the VTA TH-ir cells, the RN has enlarged and the numbers of OMs have decreased and the ON has appeared in the central grey (Fig. 1I-L).

\section{TH cell count analysis}

Using a Leica microscope (DM5000B) attached to a CCD camera (Microfire) and NeUROLUCIDA software, the number of TH-ir neurons was counted in the standard area of analysis $(700 \times 800 \mu \mathrm{m})$. Two sections from each of the rostral, middle and caudal levels of the VTA and SN were analysed and an average was calculated for each animal for each of the three rostral to caudal levels. In addition, averages across the three rostral to caudal levels were calculated for each animal. Differences between prenatal T-treated females (either T60-90 or T30-90), control males and females were compared using Kruskal-Wallis one-way ANova on ranks, Dunn's method for all pairwise multiple comparisons for the rostral VTA, and one-way ANOVA and Holm-Sidak post hoc tests for middle and caudal VTA and $\mathrm{SN}$, using $5 \%$ significance levels.

\section{Perimeter and optical density analysis}

The perimeter and optical density were analysed in the VTA of a subset of control females (C60-90; $n=7)$, males $(n=5)$ and T60 90 females $(n=5)$. For each animal, 15 images of the VTA were captured using a CCD camera (DFC 420; Leica) attached to a Leica microscope (DM5000B), using identical camera settings $(10 \times$ objective) for all images. Images were imported into IMAGEJ (National Institutes of Health, USA) and not altered in any way. For each animal, the perimeter of 45-90 TH-ir neurons (average 75) was measured and within each neuron the number of pixels above threshold were calculated as percentage of analysed area above threshold. The threshold value was calculated based on the average grey value of non-stained areas of all images and fixed for all images during analysis. For each animal, the average perimeter and average percentage of area above threshold were calculated. The group averages of T60-90 females, control males and females (C60-90) were compared using Kruskal-Wallis one-way ANova on ranks and Dunn's method with $5 \%$ significance levels.

\section{Unbiased stereology analysis}

Using STEREOINVESTIGATOR software (MicroBrightField) and the optical fractionator method, unbiased stereological estimations of the total number of TH-ir, non-TH-ir-Cresyl Violet-stained cell bodies, and total numbers of all neurons in the VTA as well as total volume of the VTA in sections from males $(n=3)$, control females (C30 $90 ; n=5)$ and T30-90 females $(n=6)$. Sections stained for $\mathrm{TH}$ with $\mathrm{DAB}$ used in the cell counting analysis described above were counterstained with Cresyl Violet and imaged using a Leica microscope (DM5000B) attached to a CCD camera (Microfire) and a motorised stage controlled by STEREOINVESTIGATOR software (MicroBrightField Inc.). The cerebral peduncle and the diameter of the AQ were used as landmarks to determine the most rostral end of the VTA and thus the location to initiate the stereological analysis. The seven subsequent sections were chosen for analysis in a rostralcaudal manner, for a total of eight tissue sections per animal. Prior to immunoprocessing, the sections were $45 \mu \mathrm{m}$ thick and every tenth parallel section was included in the analysis. Section thickness was determined by recording the distance between the plane at which the first object within the area of analysis came into focus, and the plane at which the last object went out of focus. The average final section thickness was $28.2 \mu \mathrm{m}$, accounting for shrinkage. The region of interest (i.e. the VTA) was delineated at $5 \times$ magnification. A grid measuring $700 \times 350 \mu \mathrm{m}$ was then positioned over this contour tracing; the individual counting frame used measured $190 \times 190 \mu \mathrm{m}$ with a dissector height of $10 \mu \mathrm{m}$. Individual cell bodies were identified, by an observer blinded to treatment groups, at $40 \times$ magnification, based on the identification of the nucleus of each neuron. Cells stained for $\mathrm{TH}$ with a visible nucleus were marked TH-ir, while cells stained with Cresyl Violet but not TH were marked as non-TH-ir. The optical fractionator method was used to estimate the total numbers of neurons. VTA regional volume was determined using the Cavalieri Estimator Protocol. The coefficient of error for all samples was $\leq 0.13$. One-way ANOvA and Holm-Sidak tests were used for statistical comparisons between groups. 

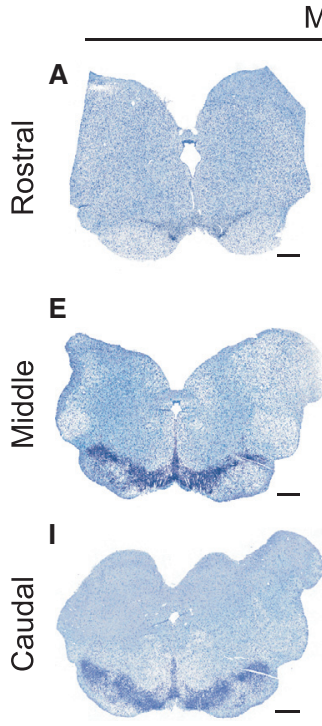

Male

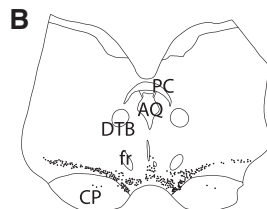

$\mathbf{F}$

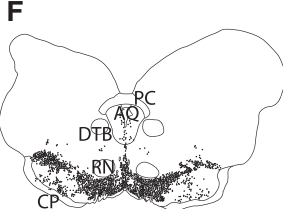

$\mathbf{J}$

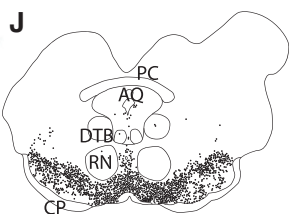

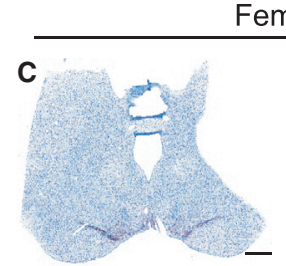

G

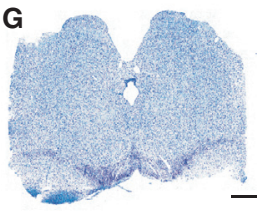

H
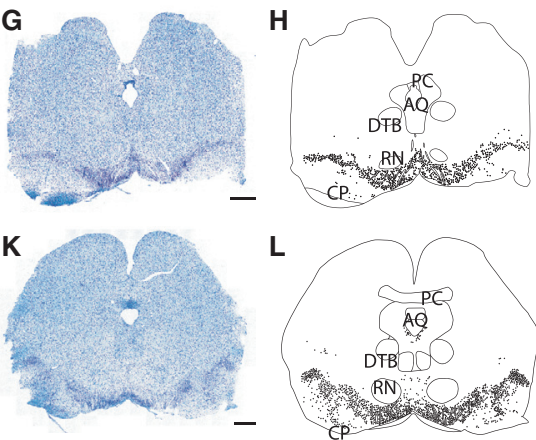

FIG. 1. Distribution of TH-ir neurons in the sheep midbrain. Representative images and contour map drawings of the male (A and B) rostral, (E and F) middle and (I and J) caudal levels, and female $(\mathrm{C}$ and $\mathrm{D})$ rostral, $(\mathrm{G}$ and $\mathrm{H})$ middle and (K and $\mathrm{L}$ ) caudal levels of the VTA and SN. Contour drawings illustrate the location of central aquaduct (AQ), dorsal tegmental bundle (DTB), fasiculus retroflexus (fr), cerebral peduncle (CP), posterior commissure (PC) and red nucleus (RN). Scale bar, $2 \mathrm{~mm}$.

\section{Photographs}

Digital images for Figures were captured using a CCD camera (DFC340FX or DFC420; Leica) attached to a Leica microscope (DM5000B). Digital images for Fig. 2 and Fig. 5 were captured using a CCD camera (Microfire) attached to a Leica microscope (DM5000B). All images were imported into ADOBE РнотоSноP software (Adobe Systems, San Jose, CA, USA) and were not altered in any way except for adjustment of brightness.

\section{Results}

\section{Characterization of the midbrain dopaminergic neurons in sheep}

There is virtually no published information about the VTA dopamine system in sheep; therefore, the neuroanatomical localization of the TH-ir neurons was first analysed in control males and females (C60-90). The distributions of TH-ir midbrain neurons in a representative male and control female are illustrated in Fig. 1. In both sexes, TH-ir neurons were observed in the midbrain, in locations corresponding to the VTA and the SN with very similar anatomical landmarks to those described in rodents (Balfour et al., 2004) (Fig. 1). Even though there were no apparent differences in the general localization of TH-ir neurons between males and females, apparent differences in TH-ir and cell number were observed (Fig. 2).

\section{Sex differences and effects of prenatal T60-90}

\section{Numbers of TH-ir cells in VTA}

Sex differences and effects of prenatal $\mathrm{T}$ were observed in the numbers of TH-ir cells in the VTA. Statistical analysis revealed an overall difference between groups at all rostral-caudal levels of the VTA $\left(F_{2,22}=31.34-33.225, P<0.001\right)$. Specifically, males had significantly greater numbers of TH-ir cells than control females (C60-90) at middle $(P<0.001)$ and caudal $(P<0.001)$ levels of the VTA (Fig. 3). Females with excess prenatal $\mathrm{T}$ treatment from GD 60-90 (T60-90 females) had significantly more TH-ir neurons than did control females at all levels of the VTA $(P<0.05,0.005,0.001$; Fig. 3) but fewer TH-ir neurons than males in the middle $(P<0.001)$ and caudal $(P=0.002)$ regions of the VTA (Fig. 3$)$.

\section{Numbers of TH-ir cells in SN}

Sex differences were also observed in $\mathrm{TH}$-ir in the $\mathrm{SN}$, as control males had significantly more cells than did control females (C60$90)$ in the middle $(P<0.001)$ and caudal $(P<0.001)$ levels, but not rostral level (Fig. 3). However, prenatal $\mathrm{T}$ treatment from GD 60-90 (T60-90) did not affect numbers of TH-ir neurons in the SN as T60-90 females were not significantly different from control females and had significantly fewer TH-ir neurons than males at the middle $(P=0.007)$ and caudal $(P=0.002)$ levels (Fig. 3).

\section{Optical density analysis of $\mathrm{TH}$-ir}

Differences between groups in intensity of $\mathrm{TH}$-ir were observed in the VTA, with more extensive dendritic and somatic labeling in males and T60-90 females than in control females (Fig. 2). Analysis of the optical density of TH-ir within the soma of VTA neurons demonstrated that males had significantly greater TH-ir optical density within the neurons than did control females $(P<0.001$; Fig. 4). T60-90 females had significantly greater TH-ir optical density in cells than did control females $(P=0.003)$, and did not differ from control males (Fig. 4). Analysis of the soma size or perimeter of TH-ir neurons in VTA revealed no significant differences between groups (Fig. 4).

\section{Effects of prenatal T30-90}

To test the effect of prenatal $\mathrm{T}$ during a longer gestational period (GD30-90), the numbers of TH-ir neurons were compared between 

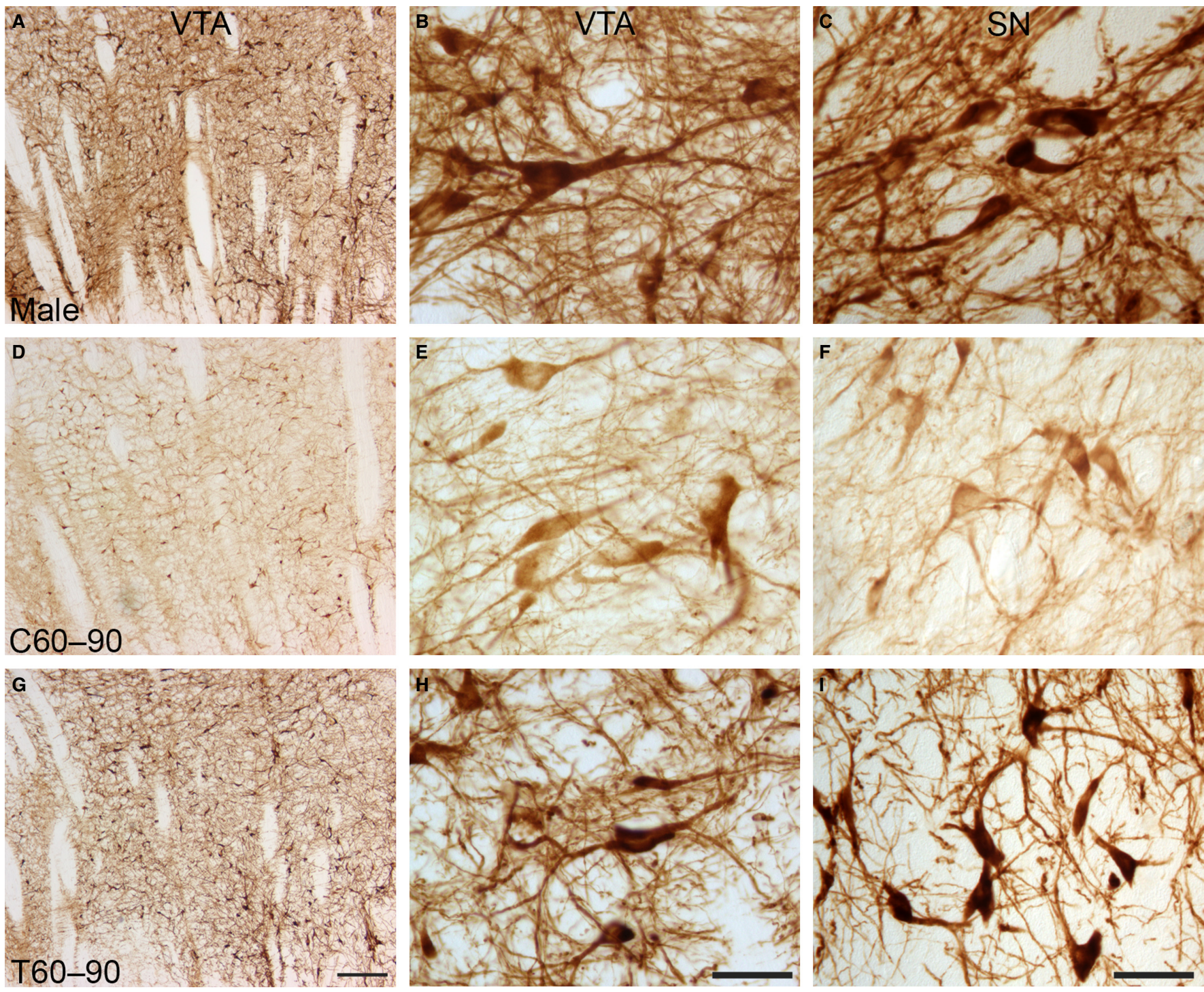

FIG. 2. Representative images of TH-ir neurons of (A-C) male, (D-F) control female (C60-90) and (G-I) prenatal T-treated female (T60-90) in the middle VTA at lower magnification (A, D and G) and higher magnification (B, E and H), and in the middle SN at higher magnification (C, F and I). Scale bars, $250 \mu \mathrm{m}$ (A, D and G), $50 \mu \mathrm{m}$ (B, C, E, F, H and I).

control males (subgroup of males in study above; the mean of VTA $\mathrm{TH}$-ir cells in this subgroup was similar to the mean of the entire group), control females (C30-90), and T30-90 females.

\section{VTA}

There was an overall difference between groups at the middle and caudal levels of the VTA, with a trend in the rostral VTA (Table 1; Rostral, $\quad F_{2,16}=3.296, \quad P=0.067 ; \quad$ Middle, $\quad F_{2,16}=15.07$, $P<0.001$; Caudal, $\left.F_{2,16}=12.49, P<0.001\right)$. As effects of prenatal $\mathrm{T}$ in the VTA were not specific to a rostrocaudal subregion (Table 1), from here on data were analysed and are presented in Fig. 5 as the average of the entire VTA (Fig. $5 ; F_{2,16}=16.51$, $P<0.001)$. Sex differences were observed and, consistent with the results described above, males had significantly greater numbers of TH-ir cells than control females $(P<0.001$, Fig. 5). T30-90 females had significantly increased numbers of TH-ir cells when compared to control females $(P<0.001)$ and did not differ from control males (Fig. 5). Moreover, differences in TH-ir were again noted within the TH-positive neurons (Fig. 5C and D) with darker immunoreactivity in T30-90 females than control females.

SN

In the $\mathrm{SN}$, sex differences were again noted at all three rostral-caudal levels (Table 2) and data are therefore also presented in Fig. 5 as an average across the three levels. Analysis showed that control males had significantly greater number of TH-ir neurons than control females $(P=0.001$; Fig. 5). In contrast, prenatal T30-90 treatment did not affect the number of TH-ir in the SN as T30-90 females did not significantly differ from control females and had significantly fewer TH-ir neurons than males $(P=0.002$; Fig. 5), confirming the results described above.

\section{Stereological analysis}

The findings thus far demonstrate that prenatal $\mathrm{T}(60-90$ or $30-90)$ increased the numbers of TH-ir neurons in the VTA and also 

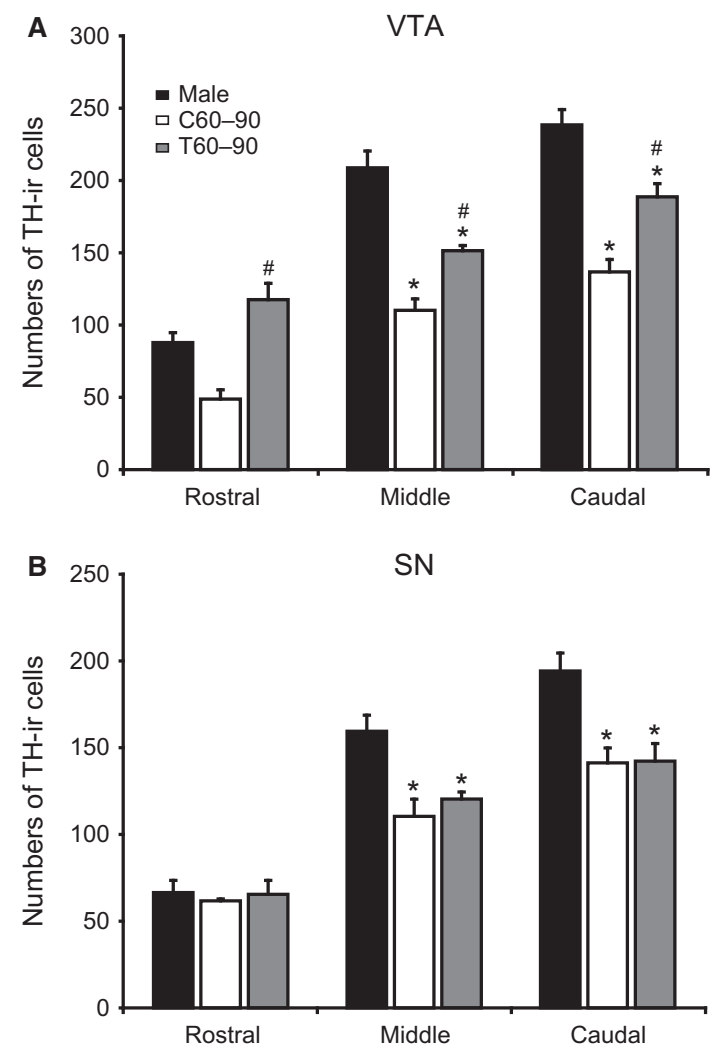

FIG. 3. Quantitative analysis of TH-ir neurons in T60-90. Mean number (+ SEM) of TH-ir cells in the rostral, middle and caudal levels of (A) the VTA and (B) the SN in control males (black; $n=8$ ), control females (C6090; white; $n=9$ ) and prenatal T-treated females (T60-90; grey, $n=6$ ). $* P<0.05$ compared to male controls; $\# P<0.05$ compared to female control C60-90 group.

increased immunoreactivity within the TH neurons, similar to males. To further test whether the sex differences and/or the effect of prenatal $\mathrm{T}$ were specific for $\mathrm{TH}$-ir cells, or were also present in nonTH-ir neurons in the VTA, a stereological analysis was conducted in control males, control females (C30-90) and T30-90 females. Secondly, it was tested whether the volume of the VTA was affected by prenatal $\mathrm{T}$ or by sex differences. Statistical analysis confirmed an overall difference in numbers of TH-ir neurons between groups $\left(F_{2,11}=26.78, \quad P<0.001\right.$; Fig. 6$)$. Specifically, control males had higher numbers of TH-ir neurons than control females $(P<0.001)$. Prenatal T-treated females $($ T30-90) had significantly more TH-ir and total numbers of cells in VTA than did control females $(P<0.001)$, and did not significantly differ from control males. In contrast, there were no sex differences or effects of prenatal T30-90 in the numbers of non-TH-ir neurons in the VTA and in the total volume of the VTA. Hence, effects of prenatal T and sex differences were specific to TH-ir neurons in the VTA. Moreover, prenatal $\mathrm{T}$ increased the total numbers of neurons in the VTA, which was mostly attributed to increases in TH-ir neurons (Fig. 6).

\section{Discussion}

The results of the present study demonstrated that the adult sheep midbrain dopamine neurons of the VTA are sexually dimorphic, with more TH-ir neurons in males than in females. Prenatal T excess during gestational days 60-90 partially masculinized the VTA dopamine system, while exposure to a longer period of prenatal $\mathrm{T}$ during
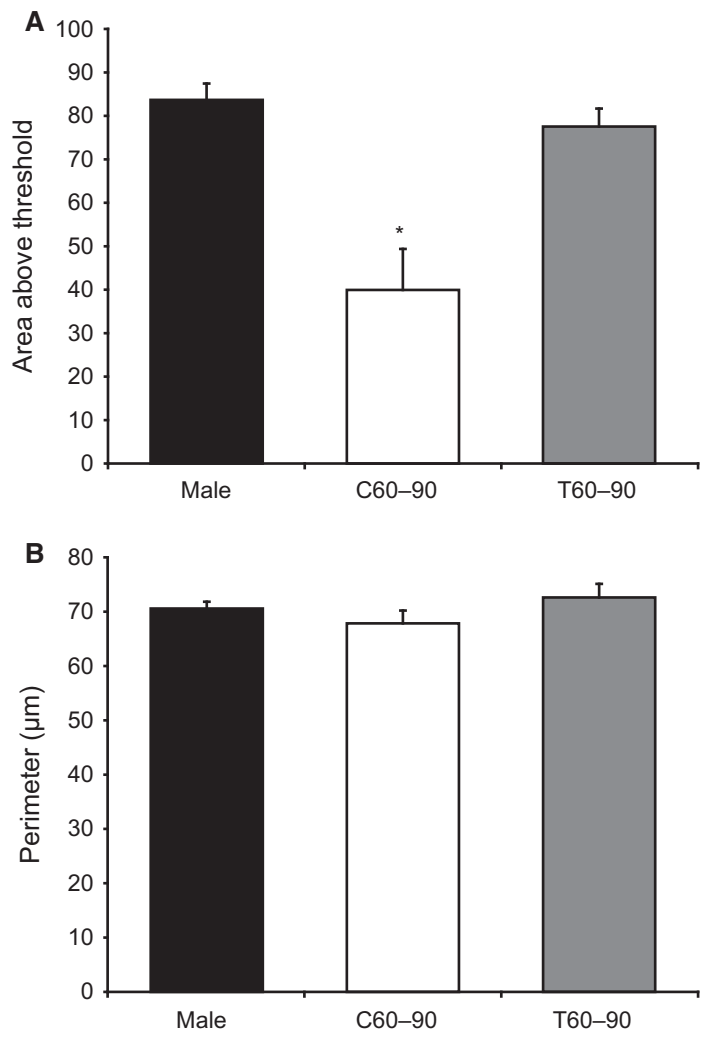

FIG. 4. TH-ir density and soma perimeter in T60-90. (A) Percentage area above threshold $(+$ SEM) of TH-ir in soma, and (B) and mean (+ SEM) perimeter of TH-ir cell bodies of control males (black; $n=5$ ), control females (C60-90; white; $n=7$ ) and prenatal T-treated females (T60-90; grey; $n=5$ ) in the VTA (A). ${ }^{*} P<0.05$ vs. males and T60-90 females.

TABLE 1. Summary of numbers of TH-ir neurons in three rostral-caudal levels in VTA

\begin{tabular}{llll}
\hline Group & Rostral & Middle & Caudal \\
\hline Male & $81.3 \pm 4.3$ & $176.5 \pm 18.3^{*}$ & $230.3 \pm 16.1^{*}$ \\
Female & $65.9 \pm 4.4$ & $106.5 \pm 6.1^{\dagger}$ & $146.9 \pm 8.9^{\dagger}$ \\
T30-90 & $84.0 \pm 8.07$ & $155.4 \pm 7.21^{*}$ & $217.7 \pm 18.0^{*}$ \\
\hline
\end{tabular}

Mean number $( \pm \mathrm{SEM})$ of TH-ir cells in the rostral, middle and caudal levels of the VTA in control males (Male; $n=4$ ), control C30-90 females (Female; $n=8$ ), prenatal T-treated females (T30-90; $n=5$ ).

*Indicates significant difference from control females $(P<0.05)$.

${ }^{\dagger}$ Indicates significant difference from male and T30-90 groups $(P<0.05)$.

gestational days 30-90 completely masculinized the VTA dopamine neurons. This was reflected by changes in both the number of dopamine neurons and the density of TH-ir. Moreover, stereological analysis showed that neither non-TH-ir cells in the VTA nor VTA volume were affected by prenatal $\mathrm{T}$. Together these results suggest that exposure to excess prenatal $\mathrm{T}$ during gestational days 30-90 results in increases in TH-ir neuron number and expression in VTA of adult ewes, mimicking sex differences in adult sheep VTA.

The findings of this study revealed a sex difference in the expression of TH-ir neurons in the VTA and SN of the sheep, with males having a significantly greater number of dopamine neurons than females in both regions. The general distributions of TH-ir neurons in the VTA and SN of male and female sheep did not differ between sexes or from those previously described in a variety of mammals, including rat (Balfour et al., 2004; Johnson et al., 2010a,b), hamster 

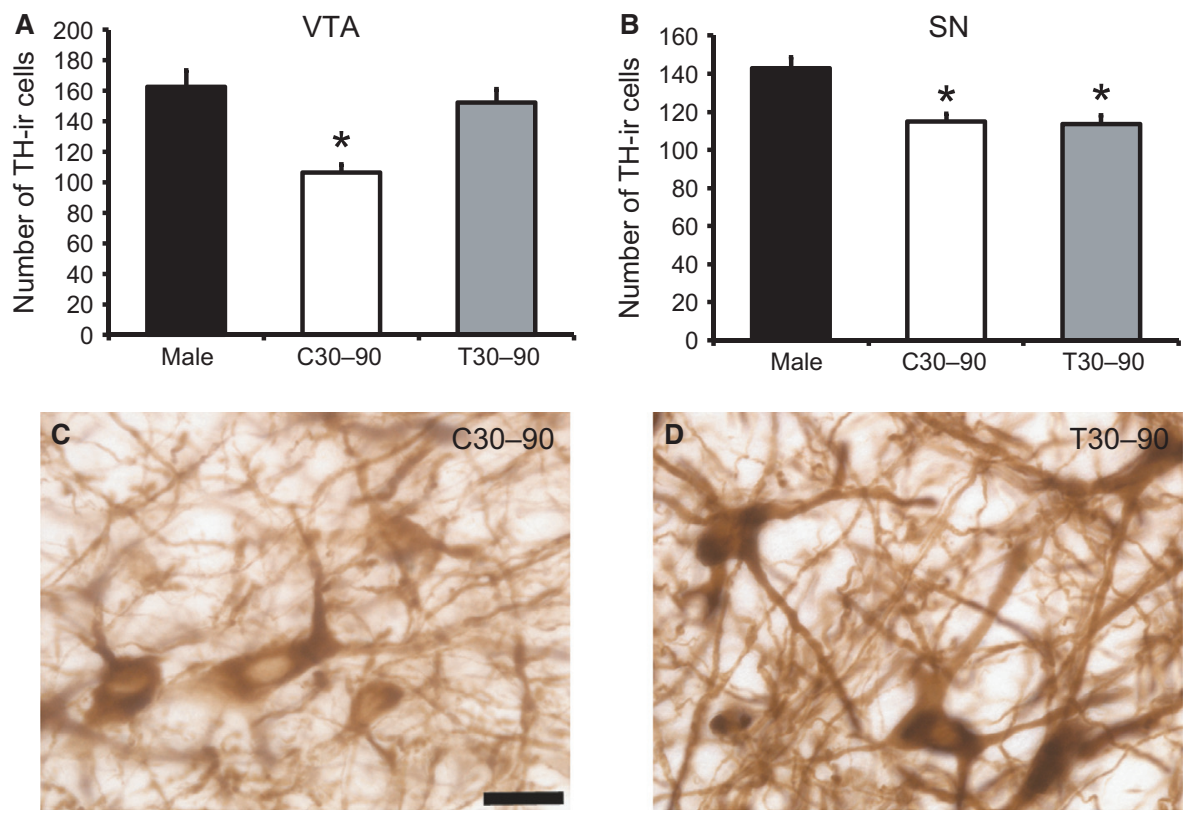

FIG. 5. Quantitative analysis of TH-ir neurons in T30-90. Mean number (+ SEM) of TH-ir cells in (A) the VTA and (B) the SN in control males $(n=4)$, control females $(\mathrm{C} 30-90 ; n=7)$ and prenatal T-treated females $(\mathrm{T} 30-90 ; n=5)$. ${ }^{*} P<0.05$ compared to male controls and T30-90 females. (C and D) Representative images of TH-immunoreactivity in VTA of control female (C30-90; C) and prenatal T-treated female (T30-90; D). Scale bar, $25 \mu \mathrm{m}$.

TABLE 2. Summary of numbers of TH-ir neurons in three rostral-caudal levels in $\mathrm{SN}$

\begin{tabular}{llll}
\hline Group & Rostral & Middle & Caudal \\
\hline Male & $66.6 \pm 1.5$ & $160.0 \pm 7.5$ & $202.0 \pm 11.0$ \\
Female & $67.7 \pm 5.3$ & $120.3 \pm 6.7^{*}$ & $156.7 \pm 6.2^{*}$ \\
T30-90 & $70.1 \pm 6.2$ & $120.7 \pm 11.6^{*}$ & $146.9 \pm 6.9^{*}$ \\
\hline
\end{tabular}

Mean number $( \pm$ SEM) of TH-ir cells in the rostral, middle and caudal levels of the SN in control males (Male; $n=4)$, control C30-90 females (Female; $n=8$ ), and prenatal T-treated females (T30-90; $n=5$ ).

*Indicates significant difference from males $(P<0.05)$.

(Vincent, 1988), pig (Ostergaard et al., 1992), sheep (Tillet \& Thibault, 1989), humans (Pearson et al., 1983) and primates (McGeer et al., 1971). However, the finding that ewes have significantly fewer TH-ir neurons in the VTA and SN than do males is in apparent contrast with previous findings in the female rat, showing significantly greater number of TH-ir neurons in the caudal VTA in females than in males (McArthur et al., 2007). Moreover, no sex differences in TH-ir neurons were detected in California Mice (Campi et al., 2013). These conflicting findings may in part be explained by species differences and/or by procedural differences in the control of steroidal milieu in the adult females. In the present study, all females were ovariectomized and received estradiol to eliminate differences in estradiol levels between groups. In contrast, the aforementioned rodent studies utilised gonadally intact females. As estradiol has been shown to increase TH-ir cells in the female rat VTA (Johnson et al., 2010b), controlling for differences in the activational effects of estradiol is critical (Arnold, 2014). However, a caveat of the present study is the lack of control for activational effects of steroids in the adult male group. Even though testosterone in male rats has been shown to cause a decrease in TH-ir neurons in the VTA (Johnson et al., 2010a), it is possible that the present finding of higher numbers of TH-ir neurons in VTA in males may be partially due to the activational actions of testosterone.
In the $\mathrm{SN}$, it has been reported that male rats have significantly greater number of dopamine neurons than females (Dewing et al., 2006; McArthur et al., 2007). Moreover, the specific expression of the Y-chromosome-linked male-determining Sry gene appears to mediate the increased expression of $\mathrm{TH}$ in the $\mathrm{SN}$, without any mediation of steroid hormones (Dewing et al., 2006). Dewing et al. demonstrated that Sry is expressed in TH-expressing neurons in the $\mathrm{SN}$ and that Sry downregulation using oligonucleotide antisense treatment caused a decrease in numbers of TH-expressing neurons in the SN. This is consistent with our findings in the sheep that males had significantly greater number of dopamine neurons in the $\mathrm{SN}$, but that prenatal $\mathrm{T}$ treatment for either 30 or 60 days did not result in increased SN dopamine neuron number in females, and suggest that the sex differences observed in the SN of the sheep may be independent of the actions of prenatal steroids and rather a result of sex chromosome complements (Arnold, 2014). However, prenatal $\mathrm{T}$ treatment in the ewe did appear to affect the expression of TH protein within SN neurons. Hence, a combination of genespecific and hormonal mediation may regulate $\mathrm{TH}$ expression and cell number in the sheep SN.

In the VTA, prenatal $\mathrm{T}$ treatment during either gestational days $30-90$ or $60-90$ resulted in a significant increase in the number of TH-ir neurons and the intensity of TH ir. However, the perimeter of the dopamine neurons was not affected by prenatal $\mathrm{T}$ exposure; this is consistent with studies in rodents, in which sex, steroid treatment or regional analysis revealed differences in TH-ir cell number but not size (McArthur et al., 2007; Johnson et al., 2010a,b). The current results suggest that the increase in the number of neurons observed in the current study in males and prenatal T-treated females is possibly a result of an increase in TH protein level expression, as demonstrated by increased levels of imunoreactivity such that more cells expressed TH at levels above the limit of detection by the immunohistochemical technique. Further evidence for this possibility arises from a study in sheep that examined effects of prenatal $\mathrm{T}$ treatment on neuropeptide expression in the arcuate nucleus of the hypothalamus. In a population of cells that co-express 
A
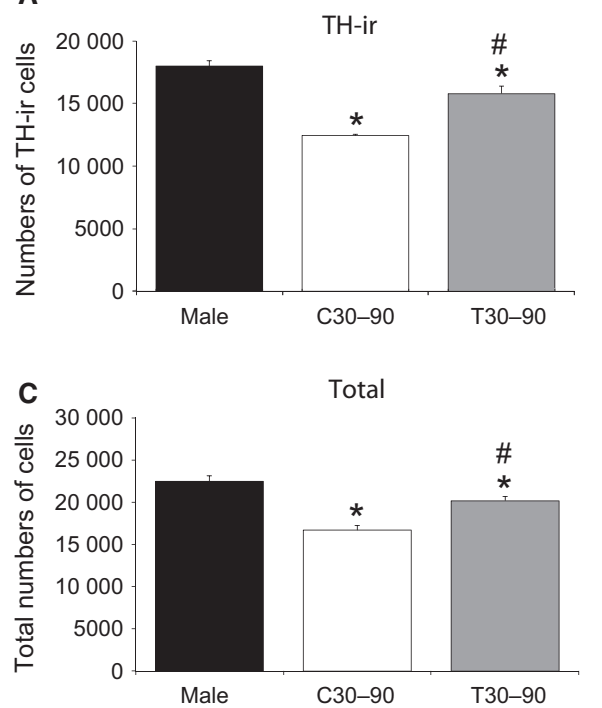

\section{B}
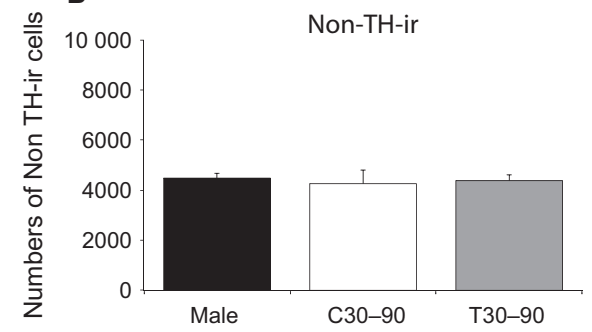

D

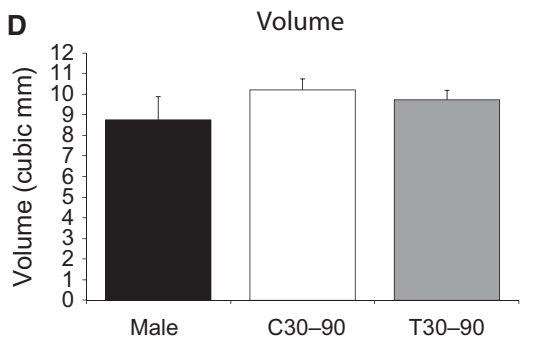

FIG. 6. Stereological analysis in T30-90. Graphs show (A) numbers of VTA TH-ir cells, (B) numbers of VTA non-TH-ir cells, (C) total numbers of VTA cells and (D) total VTA volume (in $\left.\mathrm{mm}^{3}\right)$ in control males $(N=3)$, control females $(\mathrm{C} 30-90 ; N=5)$, and prenatal T-treated females $(\mathrm{T} 30-90 ; N=6)$. Data are presented as mean + SEM. $* P<0.05$ vs. male control; $\# P<0.05$ vs. female control.

three neuropeptides: kisspeptin (KISS), neurokinin B (NKB) and dynorphin (DYN), prenatal T treatment from gestational days 30-90 resulted in a decrease in NKB and DYN; hence fewer cells expressing NKB or DYN were observed. However, the expression of KISS and the number of KISS cells was unchanged (Cheng et al., 2010). Thus, prenatal $\mathrm{T}$ did not result in cell loss per se, but rather affected expression of two out of three neuropeptides co-expressed by the same cell population. However, the results of the present study also suggest that prenatal $\mathrm{T}$ exposure alters processes of cell death and/or proliferation during development, as stereological analysis revealed an increase in total numbers of cells in prenatal T-treated females and males, which was primarily accounted for by an increase in TH-ir cells. Effects of prenatal exposure to $\mathrm{T}$ in rodents on VTA cell survival has not yet been examined, but studies in rat observed that activational effects of $\mathrm{T}$ in adulthood cause a decrease in midbrain dopamine cell survival (Johnson et al., 2010a,b).

The findings of the current study demonstrate that prenatal $\mathrm{T}$ organises the VTA dopamine system of adult sheep. It is currently unclear whether, in sheep, $\mathrm{T}$ has these organisational effects via androgenic or estrogenic actions. Previous studies in rodents have shown that $\mathrm{TH}$ gene expression can be regulated by both $\mathrm{T}$ and estradiol, which can act directly by regulating promoter activity or indirectly by altering levels of other regulator factors (Simerly et al., 1989; Thanky et al., 2002). T can be converted to DHT and act on ARs, while AR activation is able to directly increase TH expression (Jeong et al., 2006). Moreover, DHT can also be metabolised to a compound, $5 \alpha$-androstan-3 $\beta, 17 \beta$-diol, which exerts its actions through ER $\beta$ (Handa et al., 2008). Evidence from a study using the TH promoter-LacZ transgenic mouse model suggests that estradiol regulates TH expression at the transcriptional level (Thanky et al., 2002). Moreover, $T H$ transcriptional regulation by estradiol depends on the ER $\alpha$ subtype (Maharjan et al., 2005). In cultured rodent midbrain dopamine neurons, estrodiol can increase TH mRNA and protein levels (Ivanova \& Beyer, 2003) and stimulate neurite growth and plasticity (Beyer \& Karolczak, 2000). Female mice that lack the gene coding aromatase, the enzyme responsible for the conversion of $\mathrm{T}$ to estradiol, have lower SN dopamine neuron numbers than do wild-type animals (Morale et al., 2008), and removal of estradiol by ovariectomy in adult rats decreased VTA and SN dopamine neurons, an effect mediated by estrogen receptors (Johnson et al., 2010b). Thus, in rodents $\mathrm{T}$ can influence TH expression by actions on AR, ER $\beta$ or ER $\alpha$ receptors. Expression of steroid receptors in sheep VTA dopamine neurons is currently untested. However, in the rat, $\mathrm{AR}$ and ER $\beta$ are expressed in midbrain dopamine neurons (Kritzer, 1997). In rodents, ERs are present in the midbrain with ER $\beta$ having a more prominent and widespread distribution than $\mathrm{ER} \alpha$, which is not co-expressed in dopamine neurons of the VTA (Kritzer, 1997; Creutz \& Kritzer, 2002). In addition, TH neurons in VTA and SN also co-express AR (Creutz \& Kritzer, 2004; Kritzer \& Creutz, 2008). Together these results suggest that, in the rodent, sex differences in the midbrain dopamine neurons are a result of the estrogenic actions of $\mathrm{T}$. However, this remains to be determined in the sheep and will be the focus of future studies.

Together these studies demonstrate that there are sex differences in the sheep VTA dopamine system with greater numbers of TH-ir neurons in males than females. Moreover, these findings demonstrate that $\mathrm{T}$ masculinizes the VTA dopamine during prenatal life, increasing TH-ir neurons in the adult ewe. These differences in dopamine expression are the result of the organisational effects of $\mathrm{T}$ during the prenatal period, as the endogenous steroid hormones during adult life were equal in prenatal T-treated females and control females. Even though in the discussion above we have favored the hypothesis that prenatal $\mathrm{T}$ directly affected VTA dopamine neurons, we did not test whether the observed changes in the adult animal were evident immediately after birth, in early life. Hence, the possibility that prenatal T-induced changes in behavior or other functions and experiences during the animal's life could in turn influence the mesolimbic dopamine system to contribute to the observed changes cannot be eliminated and remains to be tested.

\section{Acknowledgements}

This research was supported by NIH P01 HD44232 awarded to LMC, TML, MNL and VP; NIH HD41098 awarded to VP; and NSERC PGSD to ECZB. 


\section{Abbreviations}

$\mathrm{AQ}$, central aquaduct; $\mathrm{AR}$, androgen receptor; $\mathrm{C}$, control; $\mathrm{CG}$, central grey; $\mathrm{CP}$, cerebral peduncle; DTB, dorsal tegmental bundle; ER, estrogen receptor; FR, fasciculus retroflexus; ir, immunoreactive/immunoreactivity; OM, oculomotor nerve bundle; ON, oculomotor nucleus; PB, $0.1 \mathrm{~m}$ sodium phosphate buffer; PC, posterior commissure; RN, red nucleus; SN, substantia nigra; T, testosterone; TH, tyrosine hydroxylase; VTA, ventral tegmental area.

\section{References}

Abbott, D.H., Barnett, D.K., Bruns, C.M. \& Dumesic, D.A. (2005) Androgen excess fetal programming of female reproduction: a developmental aetiology for polycystic ovary syndrome? Hum. Reprod. Update, 11, 357-374.

Alexander, B.M., Skinner, D.C. \& Roselli, C.E. (2011) Wired on steroids: sexual differentiation of the brain and its role in the expression of sexual partner preferences. Front. Endocrinol., 2, 42.

Arnold, A.P. (2014) Conceptual frameworks and mouse models for studying sex differences in physiology and disease: why compensation changes the game. Exp. Neurol., 259C, 2-9.

Balfour, M.E., Yu, L. \& Coolen, L.M. (2004) Sexual behavior and sex-associated environmental cues activate the mesolimbic system in male rats. Neuropsychopharmacology, 29, 718-730.

Beyer, C. \& Karolczak, M. (2000) Estrogenic stimulation of neurite growth in midbrain dopaminergic neurons depends on cAMP/protein kinase A signalling. J. Neurosci. Res., 59, 107-116.

Bormann, C.L., Smith, G.D., Padmanabhan, V. \& Lee, T.M. (2011) Prenatal testosterone and dihydrotestosterone exposure disrupts ovine testicular development. Reproduction, 142, 167-173.

Campi, K.L., Jameson, C.E. \& Trainor, B.C. (2013) Sexual dimorphism in the brain of the monogamous California mouse (Peromyscus californicus). Brain Behav. Evolut., 81, 236-249.

Carruth, L.L., Reisert, I. \& Arnold, A.P. (2002) Sex chromosome genes directly affect brain sexual differentiation. Nat. Neurosci., 5, 933-934.

Cheng, G., Coolen, L.M., Padmanabhan, V., Goodman, R.L. \& Lehman, M.N. (2010) The kisspeptin/neurokinin B/dynorphin (KNDy) cell population of the arcuate nucleus: sex differences and effects of prenatal testosterone in sheep. Endocrinology, 151, 301-311.

Clarke, I.J., Scaramuzzi, R.J. \& Short, R.V. (1976) Sexual differentiation of the brain: endocrine and behavioral responses of androgenized ewes to oestrogen. J. Endocrinol., 71, 175-176.

Creutz, L.M. \& Kritzer, M.F. (2002) Estrogen receptor-beta immunoreactivity in the midbrain of adult rats: regional, subregional, and cellular localization in the A10, A9, and A8 dopamine cell groups. J. Comp. Neurol., 446, 288-300.

Creutz, L.M. \& Kritzer, M.F. (2004) Mesostriatal and mesolimbic projections of midbrain neurons immunoreactive for estrogen receptor beta or androgen receptors in rats. J. Comp. Neurol., 476, 348-362.

Dewing, P., Chiang, C.W., Sinchak, K., Sim, H., Fernagut, P.O., Kelly, S., Chesselet, M.F., Micevych, P.E., Albrecht, K.H., Harley, V.R. \& Vilain, E. (2006) Direct regulation of adult brain function by the male-specific factor SRY. Curr. Biol., 16, 415-420.

Engele, J., Pilgrim, C. \& Reisert, I. (1989) Sexual differentiation of mesencephalic neurons in vitro: effects of sex and gonadal hormones. Int. J. Dev. Neurosci., 7, 603-611.

Fields, H.L., Hjelmstad, G.O., Margolis, E.B. \& Nicola, S.M. (2007) Ventral tegmental area neurons in learned appetitive behavior and positive reinforcement. Annu. Rev. Neurosci., 30, 289-316.

Frohmader, K.S., Pitchers, K.K., Balfour, M.E. \& Coolen, L.M. (2010) Mixing pleasures: review of the effects of drugs on sex behavior in humans and animal models. Horm. Behav., 58, 149-162.

Goy, R.W., Bercovitch, F.B. \& McBrair, M.C. (1988) Behavioral masculinization is independent of genital masculinization in prenatally androgenized female rhesus macaques. Horm. Behav., 22, 552-571.

Handa, R.J., Pak, T.R., Kudwa, A.E., Lund, T.D. \& Hinds, L. (2008) An alternate pathway for androgen regulation of brain function: activation of estrogen receptor beta by the metabolite of dihydrotestosterone, 5alphaandrostane-3beta,17beta-diol. Horm. Behav., 53, 741-752.

Hogg, K., McNeilly, A.S. \& Duncan, W.C. (2011) Prenatal androgen exposure leads to alterations in gene and protein expression in the ovine fetal ovary. Endocrinology, 152, 2048-2059.

Ivanova, T. \& Beyer, C. (2003) Estrogen regulates tyrosine hydroxylase expression in the neonate mouse midbrain. J. Neurobiol., 54, 638-647.
Jackson, L.M., Mytinger, A., Roberts, E.K., Lee, T.M., Foster, D.L., Padmanabhan, V. \& Jansen, H.T. (2013) Developmental programming: postnatal steroids complete prenatal steroid actions to differentially organize the GnRH surge mechanism and reproductive behavior in female sheep. Endocrinology, 154, 1612-1623.

Jeong, H., Kim, M.S., Kwon, J., Kim, K.S. \& Seol, W. (2006) Regulation of the transcriptional activity of the tyrosine hydroxylase gene by androgen receptor. Neurosci. Lett., 396, 57-61.

Johnson, M.L., Day, A.E., Ho, C.C., Walker, Q.D., Francis, R. \& Kuhn, C.M. (2010a) Androgen decreases dopamine neurone survival in rat midbrain. J. Neuroendocrinol., 22, 238-247.

Johnson, M.L., Ho, C.C., Day, A.E., Walker, Q.D., Francis, R. \& Kuhn, C.M. (2010b) Oestrogen receptors enhance dopamine neurone survival in rat midbrain. J. Neuroendocrinol., 22, 226-237.

Kritzer, M.F. (1997) Selective colocalization of immunoreactivity for intracellular gonadal hormone receptors and tyrosine hydroxylase in the ventral tegmental area, substantia nigra, and retrorubral fields in the rat. J. Comp. Neurol., 379, 247-260.

Kritzer, M.F. \& Creutz, L.M. (2008) Region and sex differences in constituent dopamine neurons and immunoreactivity for intracellular estrogen and androgen receptors in mesocortical projections in rats. J. Neurosci., 28, 9525-9535.

Maharjan, S., Serova, L. \& Sabban, E.L. (2005) Transcriptional regulation of tyrosine hydroxylase by estrogen: opposite effects with estrogen receptors alpha and beta and interactions with cyclic AMP. J. Neurochem., 93, $1502-1514$.

Manikkam, M., Crespi, E.J., Doop, D.D., Herkimer, C., Lee, J.S., Yu, S., Brown, M.B., Foster, D.L. \& Padmanabhan, V. (2004) Fetal programming: prenatal testosterone excess leads to fetal growth retardation and postnatal catch-up growth in sheep. Endocrinology, 145, 790-798.

McArthur, S., McHale, E. \& Gillies, G.E. (2007) The size and distribution of midbrain dopaminergic populations are permanently altered by perinatal glucocorticoid exposure in a sex- region- and time-specific manner. Neuropsychopharmacology, 32, 1462-1476.

McGeer, E.G., McGeer, P.L. \& Wada, J.A. (1971) Distribution of tyrosine hydroxylase in human and animal brain. J. Neurochem., 18, 1647-1658.

Merke, D.P. \& Bornstein, S.R. (2005) Congenital adrenal hyperplasia. Lancet, 365, 2125-2136.

Morale, M.C., L'Episcopo, F., Tirolo, C., Giaquinta, G., Caniglia, S., Testa, N., Arcieri, P., Serra, P.A., Lupo, G., Alberghina, M., Harada, N., Honda, S., Panzica, G.C. \& Marchetti, B. (2008) Loss of aromatase cytochrome P450 function as a risk factor for Parkinson's disease? Brain Res. Rev., 57, 431-443.

Ostergaard, K., Holm, I.E. \& Zimmer, J. (1992) Tyrosine hydroxylase and acetylcholinesterase in the domestic pig mesencephalon: an immunocytochemical and histochemical study. J. Comp. Neurol., 322, 149-166.

Padmanabhan, V., Manikkam, M., Recabarren, S. \& Foster, D. (2006) Prenatal testosterone excess programs reproductive and metabolic dysfunction in the female. Mol. Cell. Endocrinol., 246, 165-174.

Palmiter, R.D. (2007) Is dopamine a physiologically relevant mediator of feeding behavior? Trends Neurosci., 30, 375-381.

Pearson, J., Goldstein, M., Markey, K. \& Brandeis, L. (1983) Human brainstem catecholamine neuronal anatomy as indicated by immunocytochemistry with antibodies to tyrosine hydroxylase. Neuroscience, , 8, 3-32.

Roberts, E.K., Padmanabhan, V. \& Lee, T.M. (2008) Differential effects of prenatal testosterone timing and duration on phenotypic and behavioral masculinization and defeminization of female sheep. Biol. Reprod., 79, 43-50.

Roberts, E.K., Flak, J.N., Ye, W., Padmanabhan, V. \& Lee, T.M. (2009) Juvenile rank can predict male-typical adult mating behavior in female sheep treated prenatally with testosterone. Biol. Reprod., 80, 737-742.

Robinson, J.E., Birch, R.A., Foster, D.L. \& Padmanabhan, V. (2002) Prenatal exposure of the ovine fetus to androgens sexually differentiates the steroid feedback mechanisms that control gonadotropin releasing hormone secretion and disrupts ovarian cycles. Arch. Sex. Behav., 31, 35-41.

Roselli, C.E., Estill, C.T., Stadelman, H.L., Meaker, M. \& Stormshak, F. (2011) Separate critical periods exist for testosterone-induced differentiation of the brain and genitals in sheep. Endocrinology, 152, 2409-2415.

Savabieasfahani, M., Lee, J.S., Herkimer, C., Sharma, T.P., Foster, D.L. \& Padmanabhan, V. (2005) Fetal programming: testosterone exposure of the female sheep during midgestation disrupts the dynamics of its adult gonadotropin secretion during the periovulatory period. Biol. Reprod., 72, 221-229.

Serova, L.I., Maharjan, S., Huang, A., Sun, D., Kaley, G. \& Sabban, E.L. (2004) Response of tyrosine hydroxylase and GTP cyclohydrolase I gene 
expression to estrogen in brain catecholaminergic regions varies with mode of administration. Brain Res., 1015, 1-8.

Simerly, R.B., Young, B.J., Capozza, M.A. \& Swanson, L.W. (1989) Estrogen differentially regulates neuropeptide gene expression in a sexually dimorphic olfactory pathway. Proc. Natl. Acad. Sci. USA, 86, 4766-4770.

Steckler, T.L., Roberts, E.K., Doop, D.D., Lee, T.M. \& Padmanabhan, V. (2007) Developmental programming in sheep: administration of testosterone during 60-90 days of pregnancy reduces breeding success and pregnancy outcome. Theriogenology, 67, 459-467.

Thanky, N.R., Son, J.H. \& Herbison, A.E. (2002) Sex differences in the regulation of tyrosine hydroxylase gene transcription by estrogen in the locus coeruleus of TH9-LacZ transgenic mice. Brain Res. Mol. Brain Res., 104, 220-226.

Tillet, Y. \& Thibault, J. (1989) Catecholamine-containing neurons in the sheep brainstem and diencephalon: immunohistochemical study with tyrosine hydroxylase $(\mathrm{TH})$ and dopamine-beta-hydroxylase $(\mathrm{DBH})$ antibodies. J. Comp. Neurol., 290, 69-104.

Veiga-Lopez, A., Ye, W., Phillips, D.J., Herkimer, C., Knight, P.G. \& Padmanabhan, V. (2008) Developmental programming: deficits in reproductive hormone dynamics and ovulatory outcomes in prenatal, testosterone-treated sheep. Biol. Reprod., 78, 636-647.

Veiga-Lopez, A., Steckler, T.L., Abbott, D.H., Welch, K.B., MohanKumar, P.S., Phillips, D.J., Refsal, K. \& Padmanabhan, V. (2011) Developmental programming: impact of excess prenatal testosterone on intrauterine fetal endocrine milieu and growth in sheep. Biol. Reprod., 84, 87-96.

Vincent, S.R. (1988) Distributions of tyrosine hydroxylase-, dopamine-betahydroxylase-, and phenylethanolamine- $N$-methyltransferase-immunoreactive neurons in the brain of the hamster (Mesocricetus auratus). J. Comp. Neurol., 268, 584-599.

Wolf, C.J., Hotchkiss, A., Ostby, J.S., LeBlanc, G.A. \& Gray, L.E. Jr (2002) Effects of prenatal testosterone propionate on the sexual development of male and female rats: a dose-response study. Toxicol. Sci., 65, 71-86.

Wood, R.I. \& Foster, D.L. (1998) Sexual differentiation of reproductive neuroendocrine function in sheep. Rev. Reprod., 3, 130-140.

Wu, X.Y., Li, Z.L., Wu, C.Y., Liu, Y.M., Lin, H., Wang, S.H. \& Xiao, W.F. (2010) Endocrine traits of polycystic ovary syndrome in prenatally androgenized female Sprague-Dawley rats. Endocr. J., 57, 201-209. 